\title{
Relapse Prevention in Major Depressive Disorder After Successful Acute Electroconvulsive Treatment: a 6-month Double-blind Comparison of Three Fixed Dosages of Escitalopram and a Fixed Dose of Nortriptyline - Lessons from a Failed Randomised Trial of the Danish University Antidepressant Group (DUAG-7)
}

Authors

Affiliations
K. Martiny ${ }^{1}$, E. R. Larsen ${ }^{2}$, R. W. Lichtt ${ }^{3,4}$, C. T. Nielsen ${ }^{5}$, P. Damkier ${ }^{6}$, E. Refsgaard ${ }^{7}$, M. Lunde ${ }^{7}$, B. Straasø ${ }^{7}$, E. M. Christensen ${ }^{8}$, A. Lolk ${ }^{9}$, J. Holmskov9 ${ }^{\text {, C. H. Sørensen }}{ }^{9}$, I. Brødsgaard ${ }^{2}$, S. Z. Eftekhari ${ }^{10}$, B. B. Bendsen ${ }^{11}$, R. Klysner ${ }^{11}$, I. M. Terp ${ }^{10}$, J. K. Larsen ${ }^{12}$, P. Vestergaard ${ }^{2}$, P. E. Buchholtz ${ }^{2}$, L. F. Gram ${ }^{13}$, P. Bech ${ }^{7}$ and Danish University Antidepressant Group (DUAG*)

Affiliation addresses are listed at the end of the article
Key words

- electroconvulsive treatment

- major depressive disorder

relapse prevention

escitalopram

nortriptyline
Bibliography

DOI http://dx.doi.org/

$10.1055 / \mathrm{s}-0035-1565063$

Published online:

November 3, 2015

Pharmacopsychiatry 2015;

48: $274-278$

(c) Georg Thieme Verlag KG Stuttgart · New York

ISSN 0176-3679

\section{Correspondence}

\section{K. Martiny, MD, PhD}

Intensive Outpatient Unit for Affective Disorders (IAA)

Psychiatric Center Copenhagen

Rigshospitalet

Copenhagen University

Hospital

Copenhagen

Denmark

klaus.martiny@regionh.dk

\section{Abstract}

$\nabla$

Introduction: Electroconvulsive treatment (ECT) is an effective treatment for severe depression but carries a risk of relapse in the following months. Methods: Major depressive disorder patients in a current episode attaining remission from ECT (17-item Hamilton Depression Rating Scale $\left(H A M-D_{17}\right)$ score $\left.\leq 9\right)$ received randomly escitalopram $10 \mathrm{mg}, 20 \mathrm{mg}, 30 \mathrm{mg}$ or nortriptyline $100 \mathrm{mg}$ as monotherapies and were followed for 6 months in a multicentre double-blind set-up. Primary endpoint was relapse (HAM-D $17 \geq 16)$. Results: As inclusion rate was low the study was prematurely stopped with only 47 patients

\section{Introduction}

$\nabla$

Clinical practice and several randomised controlled studies have shown that a considerable proportion of patients with severe depression treated with electroconvulsive treatment (ECT) achieve remission after 8-12 treatment sessions over a period of 3-4 weeks [1]. However, the risk of relapse/recurrence is high in the following months. In a recent meta-analysis, the risk of relapse was estimated to be around $40 \%$ in a 6 month period after ECT [2]. The evidence on relapse preventing efficacy of antidepressant medication after ECT is sparse regarding choice of drugs and dosage [3].

In a randomised controlled prevention study covering a 25-week period after ECT, patients treated with paroxetine had a significantly lower risk of relapse ( $10 \%$ relapse) than patients treated with imipramine (30\% relapse) and placebo (65\% relapse) [4].

In a subsequent prevention study it was shown that $84 \%$ of subjects in remission after ECT relapsed on placebo drug treatment over a 25-week period, while treatment with nortriptyline and the combination of nortriptyline and randomised (20\% of the planned sample size). No statistically significant between-group differences could be detected. When all patients receiving escitalopram were compared with those receiving nortriptyline, a marginal superiority of nortriptyline was found $(p=0.08)$. One third of patients relapsed during the study period, and one third completed.

Discussion: Due to small sample size, no valid efficacy inferences could be made. The outcome was poor, probably due to tapering off of nonstudy psychotropic drugs after randomisation; this has implications for future study designs. ClinicalTrials.gov Identifier: NCT00660062

lithium reduced the risk of relapse to $60 \%$ and $39 \%$, respectively [5].

In the present study we aimed at investigating a potential dose-effect relationship regarding relapse prevention, by comparing daily dosages of escitalopram $10 \mathrm{mg}, 20 \mathrm{mg}$ and $30 \mathrm{mg}$, and additionally we compared these regimens with a daily dosage of $100 \mathrm{mg}$ nortriptyline, which is generally considered an effective target dose in the treatment of acute depression. A dose range was chosen for escitalopram but not for nortriptyline, since escitalopram was the primary focus, whereas nortriptyline was our reference.

Unfortunately, the planned sample size was not achieved, and therefore this report also addresses design issues having an impact on study feasibility, which might be of importance for future research in the field.

\section{Methods}

$\nabla$

\section{Organisation}

This study was carried out within the Danish University Antidepressant Group (DUAG), [6]. 


\section{Ethics and patients}

This study was carried out according to the Helsinki Declaration and the International Conference on Harmonisation-Good Clinical Practice (ICH-GCP, 1997) guideline as provided by the European Medicines Agency [7]. The Committee on Biomedical Research Ethics, the Danish Health and Medicines Authority and the Danish Central Data Register approved the study. The study was registered before start at the ClinicalTrials.gov database with identifier NCT00660062 (https://clinicaltrials.gov/ct2/ show/NCT00660062). The regional GCP units monitored the study.

Patients were screened and recruited from inpatient wards at participating psychiatric hospitals in Denmark (Hillerød, Gentofte, Rigshospitalet, Frederiksberg, Glostrup, Odense, Horsens, Esbjerg, Aarhus, and Aalborg). The patients received information, both in writing and orally, before written informed consents were obtained.

Inclusion criteria were: major depressive episode (index episode) within major depressive disorder according to DSM-IVR [8] (based on the use of the Mini International Neuropsychiatric Interview (M.I.N.I.) [9]), a completed ECT treatment, age above 18 years, a post-ECT 17-item Hamilton Depression Rating scale $\left(\right.$ HAM- $\left.\mathrm{D}_{17}\right)[10]$ score of 9 or less being present for at least 7 days, and a written informed consent.

Exclusion criteria were: suicidality corresponding to a score $>3$ on the HAM- $\mathrm{D}_{17}$ item 3 or uncertainty on the degree of suicidality as judged by the investigator, manic symptoms corresponding to a score of 15 or more on the Bech-Rafaelsen Mania scale (MAS) [10], last ECT given more than 21 days prior to planned randomisation, duration of index episode exceeding 2 years, patient under coercion, dementia or organic brain damage likely to influence the ability to give informed consent or to assess the severity of depression, a history of previous relapse occurring within 2 months after an ECT course (for ethical reasons), schizoaffective disorder, bipolar disorder, ongoing abuse of alcohol or other substances, expected low compliance with study visitschedule, treatment with fluoxetine less than 6 weeks prior to planned inclusion, current treatment with drugs being incompatible with study drugs, epilepsy, clinically significant liver or heart disease, glaucoma, pregnancy or breastfeeding, and inadequate contraception in fertile females.

A system for recording all screened and potentially eligible patients was set up at each centre.

\section{Study design}

Through a central, computerized procedure, eligible patients were randomly and double-blindly allocated to one of 4 treatment groups as described in the following with a block size of 2 [11]. The trial covered a period of 25 weeks with 16 planned data assessment points: baseline, weekly from week 1-5 and then every fortnight until week 25 with an additional safety visit at week 27. At all points patients were rated with the HAM-D ${ }_{17}$, the Hamilton Depression Rating Scale 6-item subscale HAM- $\mathrm{D}_{6}$ [12] and the MAS scale. As self-assessment scales the Major Depression Inventory (MDI) [13] and for quality of life the WHO-5 were applied [14]. Side effects were measured at all visits by the UKU scale [15].

The primary outcome was relapse defined as a HAM- $D_{17}$ scale score of 16 or above present for 14 days.
Criteria for premature study termination were: the patient wishes to withdraw consent, a MAS score higher than 15 , side effects interfering with daily activities or deemed unacceptable by investigator, protocol violation, and plasma nortriptyline levels above the chosen safety limit of $700 \mathrm{nmol} / \mathrm{l}$ at days 11 or 18 .

\section{Rater training}

As part of the set-up, investigators performed joint ratings on patients with depression using the Hamilton Depression Rating Scale. This was performed regularly at each centre, and every 4 months at a joint investigator meeting.

\section{Medication and blinding}

The dosing schedules of study medication, started after randomisation, were as follows: (Group A) $10 \mathrm{mg}$ escitalopram daily from day one and throughout the study; (Group B) $10 \mathrm{mg}$ escitalopram daily for 7 days, thereafter $20 \mathrm{mg}$ daily throughout the study; (Group C) $10 \mathrm{mg}$ escitalopram daily for 3 days, $20 \mathrm{mg}$ for 4 days and thereafter $30 \mathrm{mg}$ daily throughout the study; (Group D) $50 \mathrm{mg}$ nortriptyline for 7 days and thereafter $100 \mathrm{mg}$ daily throughout the study period. All medication was to be taken at $10 \mathrm{PM}$ in the 4 treatment groups. Medicine packages containing medication for the whole study period in the form of medication packs with 5 tablets (active and placebo tablets of identical appearance) for each day were provided. The active medications were in the form of tablets containing $10 \mathrm{mg}$ escitalopram or $50 \mathrm{mg}$ nortriptyline, respectively. The medication was produced and packaged according to GMP rules [16] by a contract research organisation [11].

All concomitant drugs were tapered off over a maximum period of 8 weeks after randomisation. At the beginning of the study the tapering period was set to 2 weeks but due to observed discontinuation symptoms and many early relapses, we extended the tapering period to 8 weeks after 15 patients had been included. Hypnotics in recommended dosage and benzodiazepines up to a dosage corresponding to a daily dosage of $45 \mathrm{mg}$ oxazepam were allowed throughout the whole study period. Blood samples for plasma monitoring of escitalopram and nortriptyline were drawn in the morning on days 11 and 18 [17].

\section{Sample size estimation}

With a hypothesized risk of relapse over a 25-week study period of $30 \%$ for patients treated with $10 \mathrm{mg}$ escitalopram daily, it would require 60 patients in each group (and a total number of 240 patients) to detect a $20 \%$ difference between $30 \mathrm{mg}$ escitalopram and $10 \mathrm{mg}$ escitalopram and between $30 \mathrm{mg}$ escitalopram daily and $100 \mathrm{mg}$ nortriptyline daily, at the $5 \%$ level of statistical significance with a power of $80 \%$.

\section{Data analysis}

This is a Phase IV trial performed to test any difference between the 4 treatment groups with relapse rates as the primary outcome. All randomised patients assessed at one or more postbaseline visits were included in analyses (modified intention to treat population). Based on time to the relapse, a survival analytical approach was used with Kaplan-Meier curves and log rank test. In the survival analyses, observations that were terminated for other reasons than the endpoint in question were censored.

In a post-hoc analysis, all patients receiving escitalopram were compared with those receiving nortriptyline. In another post- 


\begin{tabular}{|c|c|c|c|c|c|}
\hline Characteristic & $\begin{array}{c}\text { Escitalopram } \\
\begin{array}{c}10 \mathrm{mg} \\
\mathrm{n}=11\end{array}\end{array}$ & $\begin{array}{l}\text { Escitalopram } \\
\begin{array}{c}20 \mathrm{mg} \\
\mathrm{n}=11\end{array}\end{array}$ & $\begin{array}{c}\text { Escitalopram } \\
\begin{array}{c}30 \mathrm{mg} \\
\mathrm{n}=10\end{array}\end{array}$ & $\begin{array}{l}\text { Nortriptyline } \\
\begin{array}{c}100 \mathrm{mg} \\
n=14\end{array}\end{array}$ & $\mathbf{P}$ \\
\hline Age, years mean (SD) & $54.1(20.3)$ & $56.5(10.8)$ & $54.6(13.5)$ & $55.8(13.8)$ & 0.99 \\
\hline Female gender, per cent & $72.7 \%$ & $36.4 \%$ & $40.0 \%$ & $85.7 \%$ & 0.03 \\
\hline Numb. episode mean (SD) & $3.7(3.3)$ & $1.1(1.1)$ & $2.1(1.8)$ & $3.5(5.5)$ & 0.29 \\
\hline Dur. depres. illness, years, mean (SD) & $22.3(14.1)$ & $10.4(11.8)$ & $10.6(13.8)$ & $9.2(13.7)$ & 0.10 \\
\hline Duration episode, months, mean (SD) & $9.4(11.7)$ & $10.5(7.2)$ & $5.5(4.1)$ & $6.2(2.9)$ & 0.36 \\
\hline HAM- ${ }_{17}$ inclusion (SD) & $6.5(2.5)$ & $5.8(2.5)$ & $4.2(2.7)$ & $5.6(2.9)$ & 0.25 \\
\hline HAM-D ${ }_{6}$ inclusion (SD) & $3.7(1.7)$ & $3.7(2.2)$ & $2.6(2.0)$ & $2.9(2.1)$ & 0.42 \\
\hline MAS inclusion (SD) & $0.5(0.8)$ & $0.5(1.5)$ & $0.6(1.0)$ & $0.1(0.4)$ & 0.68 \\
\hline MDI inclusion (SD) & $10.6(6.0)$ & $16.8(7.7)$ & $8.9(8.2)$ & $12.8(9.1)$ & 0.12 \\
\hline WHO-5 inclusion (SD) & $64.4(16.7)$ & $54.9(24.6)$ & $61.6(25.8)$ & $65.4(28.1)$ & 0.79 \\
\hline SSRI & 1 & 3 & 1 & 4 & \\
\hline SNRI & 3 & 6 & 4 & 3 & \\
\hline Mirtazapine & 4 & 0 & 3 & 4 & \\
\hline Tricyclic & 0 & 2 & 1 & 3 & \\
\hline Quetiapine & 2 & 2 & 3 & 1 & \\
\hline Olanzapine & 0 & 4 & 1 & 2 & \\
\hline Chlorprothixene & 0 & 2 & 0 & 1 & \\
\hline Risperidone & 0 & 0 & 1 & 0 & \\
\hline Flupentixol & 0 & 0 & 0 & 1 & \\
\hline Lithium & 0 & 0 & 0 & 0 & \\
\hline Other & 0 & 3 & 1 & 3 & \\
\hline
\end{tabular}

Table 1 Baseline characteristics with standard deviations in brackets. hoc pooled analysis of all patients receiving escitalopram, patients were divided according to the median escitalopram concentration. Survival analysis, using relapse as outcome, was then performed on the group of patients with concentrations respectively above and below the median to investigate any influence of plasma concentration on relapse.

\section{Results}

$\nabla$

More than 600 patients were screened for inclusion in the study, but due to the multiple exclusion criteria, patients' disinclination to participate and clinicians' reluctance to refer patients to the trial, recruitment was slow. Therefore the study was prematurely stopped when 47 patients had been randomised, i.e., $20 \%$ of the planned sample size. All patients entered the study from September 2009 to November 2012. One patient was withdrawn before drug administration due to early relapse and was not included in the efficacy analysis. Inter-rater reliability for training sessions was very high with an intraclass coefficient (ICC) of 0.93 for HAM- $\mathrm{D}_{17}$, and 0.89 for HAM- $\mathrm{D}_{6}$, and 0.91 for MES.

- Table 1 shows sociodemographics, baseline psychometric values, and psychotropic drug use. Gender ratios differed between groups with more females in the escitalopram $10 \mathrm{mg}$ and nortriptyline groups $(p=0.03)$. We analysed the number of completers vs. the number of relapses for females/males and found no statistically significant relation between outcome and gender $(p=0.46)$. No data were available on patients' HAM-D scores prior to their ECT course.

- Table 2 shows patient disposition (endpoint all causes) and number of patients treated with different psychotropic drugs at baseline for each group. Overall, 16 (35\%) patients relapsed and 15 (33\%) had a successful outcome. 4 patients dropped out due to expiration of medication (administrative) due to delay in deliverance of a new medication batch from the contract research organisation. 2 patients had to be taken out due to serious adverse events (SAE): one patient treated with $10 \mathrm{mg}$ escit-
Table 2 Patient disposition (endpoint all causes).

\begin{tabular}{|c|c|c|c|c|c|}
\hline Outcome & $\begin{array}{l}\text { Escitalo- } \\
\text { pram } \\
10 \mathrm{mg}\end{array}$ & $\begin{array}{l}\text { Escitalo- } \\
\text { pram } \\
20 \mathrm{mg}\end{array}$ & $\begin{array}{l}\text { Escitalo- } \\
\text { pram } \\
30 \mathrm{mg}\end{array}$ & $\begin{array}{l}\text { Nortrip- } \\
\text { tyline } \\
100 \mathrm{mg}\end{array}$ & Total \\
\hline Completer & 3 & 5 & 1 & 6 & 15 \\
\hline Relapse & 5 & 4 & 5 & 2 & 16 \\
\hline $\begin{array}{l}\text { Left due to } \\
\text { side effect }\end{array}$ & 0 & 0 & 1 & 0 & 1 \\
\hline $\begin{array}{l}\text { Left due to } \\
\text { high plasma } \\
\text { conc. }\end{array}$ & 0 & 0 & 0 & 3 & 3 \\
\hline $\begin{array}{l}\text { Wanted to } \\
\text { end study }\end{array}$ & 2 & 1 & 2 & 1 & 6 \\
\hline $\begin{array}{l}\text { Protocol } \\
\text { violation }\end{array}$ & 1 & 0 & 0 & 0 & 1 \\
\hline Administrative & 0 & 1 & 1 & 2 & 4 \\
\hline Total & 11 & 11 & 10 & 14 & 46 \\
\hline
\end{tabular}

alopram was re-admitted due to insomnia, probably caused by tapering-off of usual medication, and one patient treated with $30 \mathrm{mg}$ escitalopram was readmitted to hospital due to prolongation of the ECG QTC interval above recommended value. 3 patients had to be taken out due to plasma nortriptyline concentrations above the stated safety limit of $700 \mathrm{nmol} / 1$ (one patient with $801 \mathrm{nmol} / \mathrm{l}$ at day 11 , one patient with $1182 \mathrm{nmol} / \mathrm{l}$ at day 18 , and one patient with $1090 \mathrm{nmol} / \mathrm{l}$ at day 18). Only one patient had plasma concentration below the recommended efficacy limit of $230 \mathrm{nmol} / \mathrm{l}$ on both days.

- Fig. 1 shows Kaplan-Meier curves, based on relapse, for all 4 groups. No overall statistically significant difference could be detected (log-rank test, $\mathrm{p}=0.10$ ).

When all patients treated with escitalopram were compared with the nortriptyline treated patients, numerically, the nortriptyline group performed better and this approached statistical significance (log-rank test, $\mathrm{p}=0.08$ ) ( $\bullet$ Fig. 2). No associations between escitalopram plasma concentration and risk of relapse were found $(\mathrm{p}=0.83)$. 


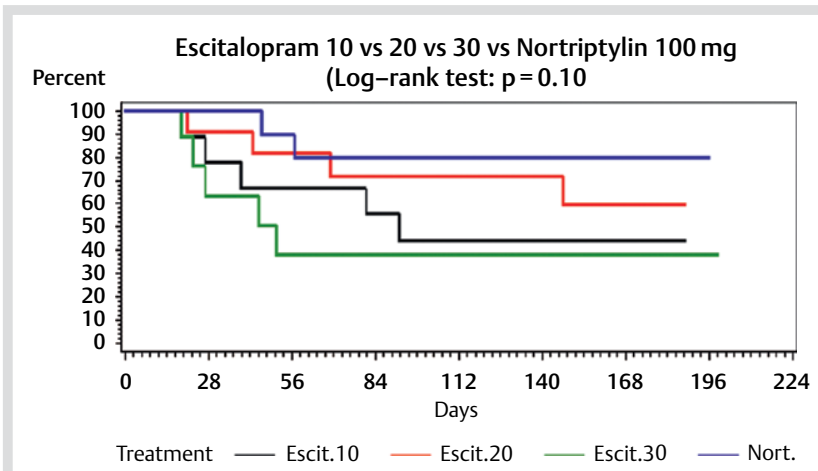

Fig. 1 Kaplan-Meier curves for the 4 treatment groups based on relapse.

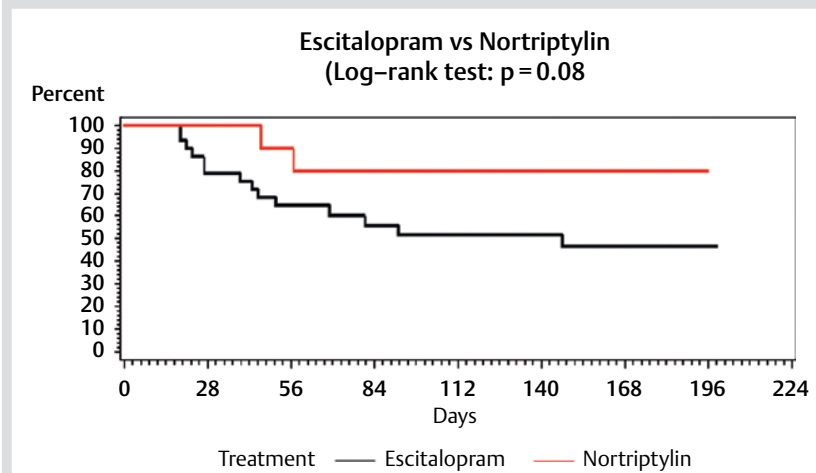

Fig. 2 Kaplan-Meier curves for all patients receiving escitalopram (10 $\mathrm{mg}$ or $20 \mathrm{mg}$ or $30 \mathrm{mg}$ ) and the nortriptyline group based on relapse.

Results on all-cause endpoints were similar to the above results (data not shown).

Regarding the UKU side-effect scale, the sum scores (last observation carried forward) were low and without statistically significant differences between groups.

\section{Discussion}

$\nabla$

The most important finding from this study is that one third of patients relapsed over the 6-month study period across all 4 treatment arms, and only one third had a successful outcome. This poor overall outcome is comparable with that of Sackeim et al [5]. Our study outcome might have been more favourable if patients had been started on pharmacotherapy before or at the same time as commencement of ECT as discussed below.

In this study we could not demonstrate statistically significant differences in risk of relapse between the 4 interventions. The lack of discrimination between risks of relapse in the 4 groups might obviously be ascribed to the small sample size (type II error). It could also be a true negative finding relating to the discontinuation of non-study drugs in all treatment groups. Relapse due to per-protocol tapering-off of non-study medication was also observed in a recently published long-term study from the DUAG [18]. However, it is worthwhile noting that patients treated with nortriptyline had a lower risk of relapse than the pooled group of patients treated with escitalopram, on an $8 \%$ statistical significance level. The nortriptyline dosage seemed to be adequate as only one patient had a level below the recommended plasma concentration efficacy level (Danish Medicines Agency). Even though the marginal superiority of nortriptyline obviously might be related to the inclusion of patients dosed relatively low in the pooled escitalopram group, i.e., $10 \mathrm{mg}$, nortriptyline arm performance was numerically superior to each of the escitalopram arms ( $\triangle$ Table 2 ). We found no association between escitalopram plasma concentrations and the risk of relapse, although a recent study indicated a relationship between plasma citalopram and antidepressant efficacy [19]. Our negative finding on that aspect might be ascribed to the small sample size.

The results from the WHO-5 well-being scale confirm previous results showing that patients in remission, as defined by the HAM- $D_{17}$ scale, do still have some degree of poor well-being [20], below the Danish population mean of 68.7 [21] which emphasises the need for a broader concept of remission that includes patient perspective and side effects.

Our experience from this multicentre study is that it was difficult to recruit patients to a relapse prevention study when using mono-therapy after ECT. During the study period it became clear that the majority of patients had received one or more medications during ECT, and that the tapering-off of such ongoing non-study medications after randomisation caused distress and probably in itself increased risk of relapse. Accordingly, and based on impressions from regular consultations with centres, clinicians were reluctant to refer these severely ill hospitalised patients to a post-ECT trial using only mono-therapy study drugs. Unfortunately, the planned registration of all screened patients was incomplete, resulting in that no valid data on the distribution of eligible patients on various reasons for non-randomisation were available. Besides potentially reducing the power of a trial, non-randomisation of eligible patients, whether due to patients' or due to clinicians' resistance will most likely lead to limitations in generalisability of the study results [22].

Future studies should take the issues outlined above into consideration by allowing co-medication after randomisation. Another approach would be to start study medication prior to ECT, thereby maybe reducing the risk of early relapse and making the design more acceptable for patients and clinicians. This is supported by the study by Yildiz et al. [23] who found a significant reduction in relapse when trial medication was started early compared to later in the ECT course but not by the study by Prudic et al. [24], who found no difference in relapse rates when pharmacotherapy was started before or after ECT. However, if patients are randomised before remission has been obtained, the outcome of the post-randomisation acute treatment needs to be taken into account.

Overall, one third of patients relapsed over the 6-month study period, and only one third had a successful outcome. This calls for more research in this area.

The primary limitation was the small sample size with only $20 \%$ reached, in spite of allocation of patients for a prolonged period at 10 centres.

The main lesson to be learned is that allocating patients to study drugs given as monotherapy after remission is not feasible, and that pilot studies carefully revealing the reasons for non-inclusion of eligible patients are necessary [25]. 


\section{Acknowledgements}

$\nabla$

An unrestricted grant was provided from Lundbeck A/S and from the Danish Agency for Science, Technology and Innovation. We thankfully acknowledge the following persons for support throughout all stages of the project: Lotte Hjerrild Buxbom and Claus Zander Jensen (Risskov) Line Cramer, Line Møller og Peter Thorsbøll (Gentofte) Heidi Borup (Aalborg) Anne-Lene Kjeldmann, Piotr Zbigniew Schulz, and Katarzyna Schulz (Esbjerg) Lars Djärv (Lundbeck)

\section{Conflict of Interest}

$\nabla$

Klaus Martiny and Else Refsgaard had part of their salary paid from a Lundbeck grant. Investigators received a grant for the inclusion of patients. This grant was solely to be used for research purposes and research education. No authors indicated any other conflicts of interest regarding the submitted paper.

\section{Affiliations}

Intensive Outpatient Unit for Affective Disorders (IAA), Psychiatric Center

Copenhagen, Rigshospitalet, Copenhagen University Hospital, Copenhagen, Denmark

${ }^{2}$ Department of Affective Disorders Mood Disorders Research Unit, Aarhus University Hospital, Aarhus, Denmark

${ }^{3}$ Department of Clinical Medicine, Aalborg University, Aalborg, Denmark

Aalborg University Hospital, Psychiatry, Aalborg, Denmark

Department of Mental Health Services, Esbjerg, Denmark

${ }^{6}$ Department of Clinical Chemistry \& Pharmacology, Odense University Hospital, Odense, Denmark

Psychiatric Research Unit, Mental Health Centre North Zealand, University of Copenhagen, Copenhagen, Denmark

${ }^{8}$ The Mood Disorder Clinic, Psychiatric Center Copenhagen, Rigshospitalet, Copenhagen University Hospital, Copenhagen, Denmark

${ }^{9}$ Department of Psychiatry, Odense University Hospital, Odense, Denmark

${ }^{10}$ Psychiatric Center Glostrup, Copenhagen University Hospital, Copenhagen, Denmark

${ }^{11}$ Psychiatric Center Frederiksberg, Copenhagen University Hospital, Copenhagen, Denmark

${ }^{12}$ Psychiatric Center Gentofte, Copenhagen University Hospital, Copenhagen, Denmark

${ }^{13}$ Clinical Pharmacology, Institute of Public Health, University of Southern Denmark, Odense, Denmark

\section{References}

1 Taylor S. Electroconvulsive therapy: a review of history, patient selection, technique, and medication management. South Med J 2007; 100 : 494-498

2 Jelovac A, Kolshus E, McLoughlin DM. Relapse following successful electroconvulsive therapy for major depression: a meta-analysis. Neuropsychopharmacology 2013; 38: 2467-2474

3 Youssef NA, McCall WV. Relapse prevention after index electroconvulsive therapy in treatment-resistant depression. Ann Clin Psychiatry 2014; 26: 288-296

4 Lauritzen L, Odgaard $K$, Clemmesen $L$ et al. Relapse prevention by means of paroxetine in ECT-treated patients with major depression: a comparison with imipramine and placebo in medium-term continuation therapy. Acta Psychiatr Scand 1996; 94: 241-251

5 Sackeim HA, Haskett RF, Mulsant BH et al. Continuation pharmacotherapy in the prevention of relapse following electroconvulsive therapy: a randomized controlled trial. JAMA 2001; 285: 1299-1307
6 DUAG Homepage [homepage on the Internet]: DUAG; 2015; Available from: www.duag.dk

7 EMEA. The European Agency for the Evaluation of Medicinal Products. Note for Guidance on Good Clinical Practice (CPMP/ICH/135/95). London: EMEA; 1997

8 American Psychiatric Association. The Diagnostic and Statistical Manual of Mental Disorders. Fourth Edition (DSM-IV).Washington D.C.: American Psychiatric Association; 1994

9 Sheehan DV, Lecrubier Y, Sheehan KH et al. The Mini-International Neuropsychiatric Interview (M.I.N.I.): the development and validation of a structured diagnostic psychiatric interview for DSM-IV and ICD-10. J Clin Psychiatry 1998; 59 (Suppl 20): 22-33

10 Bech P. Clinical Psychometrics. Oxford: Wiley Blackwell; 2012

11 Almac Group [homepage on the Internet]. Available from: http://.com/ almacgroup.com

12 O'Sullivan RL, Fava M, Agustin C et al. Sensitivity of the six-item Hamilton Depression Rating Scale. Acta Psychiatr Scand 1997; 95: 379-384

13 Bech P, Rasmussen NA, Olsen LR et al. The sensitivity and specificity of the Major Depression Inventory, using the Present State Examination as the index of diagnostic validity. J Affect Disord 2001; 66: 159-164

14 Bech P. Measuring the dimension of Psychological General Well-Being by the WHO-5. Quality of Life Newsletter (Mapi Research Institute) 2004; 32: 15-16

15 Lingjaerde 0 , Ahlfors UG, Bech P et al. The UKU side effect rating scale. A new comprehensive rating scale for psychotropic drugs and a crosssectional study of side effects in neuroleptic-treated patients. Acta Psychiatr Scand Suppl 1987; 334: 1-100

16 EudraLex. Volume 9, Good Manufacturing Practices (GMP) ec.europa. eu/health/files/eudralex/vol-9/pdf/vol9_10-2004_en.pdf ed. European Commission

17 Vuille F, Amey M, Baumann P. Use of plasma level monitoring of antidepressants in clinical practice. Towards an analysis of clinical utility. Pharmacopsychiatry 1991; 24: 190-195

18 Licht RW, Danish University Antidepressant Group. Is it possible to evaluate true prophylactic efficacy of antidepressants in severely ill patients with recurrent depression? Lessons from a placebo-controlled trial. The fifth trial of the Danish University Antidepressant Group (DUAG-5). J Affect Disord 2013; 148: 286-290

19 Ostad Haji E, Tadic A, Wagner $S$ et al. Early improvement and serum concentrations of citalopram to predict antidepressant drug response of patients with major depression. Pharmacopsychiatry 2013; 46: 261-266

20 Zimmerman M, Martinez JA, Attiullah $N$ et al. Why do some depressed outpatients who are in remission according to the Hamilton Depression Rating Scale not consider themselves to be in remission? J Clin Psychiatry 2012; 73: 790-795

21 Bech $P$, Olsen LR, Kjoller $M$ et al. Measuring well-being rather than the absence of distress symptoms: A comparison of the SF-36 Mental Health subscale and the WHO-Five Well-Being Scale. Int J Methods Psychiatr Res 2003; 12: 85-91

22 Bliznak L, Berg $R$, Hage A et al. High rate of non-eligibility: methodological factors impacting on recruitment for a multicentre, doubleblind study of paediatric patients with major depressive disorder. Pharmacopsychiatry 2013; 46: 23-28

23 Yildiz A, Mantar A, Simsek $S$ et al. Combination of pharmacotherapy with electroconvulsive therapy in prevention of depressive relapse: a pilot controlled trial. J ECT 2010; 26: 104-110

24 Prudic J, Haskett RF, McCall WV et al. Pharmacological strategies in the prevention of relapse after electroconvulsive therapy. J ECT 2013; 29: 3-12

25 Arain M, Campbell MJ, Cooper CL et al. What is a pilot or feasibility study? A review of current practice and editorial policy. BMC Med Res Methodol 2010; 10: 67-2288-10-67 